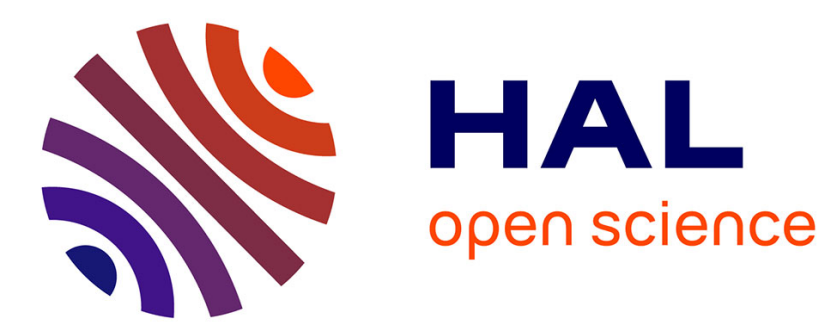

\title{
L'Identification des Idées
}

Vincent Descombes

\section{To cite this version:}

Vincent Descombes. L'Identification des Idées. Revue philosophique de Louvain, 1998, 91 (1), pp.86118. ijn_00000512

\section{HAL Id: ijn_00000512 \\ https://hal.science/ijn_00000512}

Submitted on 11 Jun 2004

HAL is a multi-disciplinary open access archive for the deposit and dissemination of scientific research documents, whether they are published or not. The documents may come from teaching and research institutions in France or abroad, or from public or private research centers.
L'archive ouverte pluridisciplinaire HAL, est destinée au dépôt et à la diffusion de documents scientifiques de niveau recherche, publiés ou non, émanant des établissements d'enseignement et de recherche français ou étrangers, des laboratoires publics ou privés. 


\section{Vincent Descombes \\ L'IDENTIFICATION DES IDÉES}

Résumé

L'intérêt considérable de la théorie de la culture défendue par Dan Sperber, dans son livre La contagion des idées, est d'expliciter plus fermement que de coutume les présupposés ontologiques d'une approche atomiste dans ce domaine. L'ethnographe présuppose qu'il y a dans le monde, non seulement des personnes, mais des idées. Comment les idées sont-elles dans le monde ? Le "monisme ontologique" de Sperber consiste à refuser de multiplier les genres d'entités. Les idées sont donc, selon lui, des entités matérielles au même titre que les personnes. Pourtant, le véritable problème ontologique qui se pose au sujet des idées n'est pas de décider si elles sont des objets matériels ou plutôt des objets immatériels, mais de savoir si leur mode d'être est celui des objets ou s'il est d'une autre catégorie que celle des objets.

\section{Le paradoxe de la communication précaire}

Certaines philosophies du langage ont l'inconvénient de comporter une conséquence paradoxale : à la lumière de leurs prémisses, les faits ordinaires de communication prennent un petit air d'événements improbables, pour ne pas dire de miracles. La communication par signes serait foncièrement précaire.

Soit l'exemple suivant d'un échange verbal ordinaire :

— Un passant inconnu s'adresse à moi et me demande : Y a-t-il une boulangerie ouverte le lundi dans ce quartier?

— Moi : Oui, dans la prochaine rue à droite.

— Le passant : Merci !

$\mathrm{Au}$ cours de cet échange, je donne une réponse à quelqu'un que je ne connais ni d'Eve ni d'Adam, dont je ne connais ni les opinions ni les idées. J'ai donc fait comme si je pouvais aisément donner un sens aux mots qu'il emploie et à la construction interrogative dans laquelle il les a utilisés.

On peut faire légèrement varier l'exemple. C'est maintenant moi qui pose la question, c'est l'inconnu qui répond, et c'est donc moi qui le remercie. Dans ce cas, je m'adresse à un inconnu comme si cet inconnu pouvait me répondre. Or il se trouve que, bien souvent, cet inconnu me répond, comme s'il trouvait naturel de m'avoir compris et, qui plus est, sans paraître douter un seul instant que ses paroles, à leur tour, seront comprises par moi. De temps à autre, il est vrai (par exemple, dans certains quartiers du centre de Paris), l'inconnu se découvre être un touriste qui ne sait pas un mot de français. Dans ces derniers cas (qui ne nous paraissent d'ailleurs nullement surprenants), nous nous bornons à enregistrer un échec à engager la conversation. 
Est-ce qu'il y aurait une difficulté fondamentale dissimulée dans les "comme si" de ces exemples?

D'après plusieurs philosophes contemporains, les échecs à communiquer ne se produisent pas seulement dans ces circonstances spéciales où un touriste s'adresse à moi dans une langue que je ne comprends pas, ou bien dans celles où le passant à qui je demande un renseignement n'est pas en mesure de m'entendre. A côté de ces cas triviaux d'incompréhension, il pourrait y en avoir d'autres, moins faciles à détecter et par là plus instructifs pour le philosophe. Deux personnes se parlent. Elles paraissent se comprendre. En apparence, la communication a lieu. Pourtant, nous aurions des raisons philosophiques de nous demander si elles se comprennent réellement, si la communication dans laquelle elles semblent engagées ne repose pas sur un malentendu.

Deux arguments ont été récemment avancés qui entraînent cette conséquence d'un soupçon pesant sur le principe même d'une communication entre les humains. Le premier argument exploite les conséquences d'une thèse holiste en philosophie du langage : toute communication serait précaire dès lors que l'assignation du sens ne se fait pas aux signes pris les uns après les autres, chacun pour soi, mais à une totalité linguistique. Le second argument résulte, à l'opposé, d'une thèse fermement individualiste : toute communication est précaire, nous est-il suggéré, parce que les signes qu'utilise mon interlocuteur pour communiquer la pensée qu'il a en tête ne sont pas à proprement parler l'expression de sa pensée, mais la traduction de celle-ci en signes publics (ils sont la "représentation" publique d'une "représentation" privée); pour que la communication ait lieu, il me faut construire une version (privée) de ce qui est signifié par son discours (public), et donc fatalement interpréter, à mes risques et mes périls, ses paroles.

Soit d'abord le premier de ces arguments. Dans l'ouvrage qu'ils ont écrit contre les conceptions holistes du langage et de l'esprit ${ }^{1}$, Fodor et LePore soutiennent que le holisme sémantique (qui est, notent-ils, la conception aujourd'hui dominante) a pour conséquence de mettre en doute la réalité de la communication sous toutes ses formes. Bien entendu, c'est là pour eux une puissante objection contre toute théorie de ce genre.

Fodor et LePore distinguent utilement deux versions possibles du holisme. Ils réservent l'étiquette de "holisme anthropologique" aux philosophies qui, à l'exemple du second Wittgenstein, posent que les propriétés sémantiques des signes sont fonction d'un contexte humain, social et historique. Le holisme consiste ici à réclamer que le sens des signes soit expliqué par leur usage dans un contexte anthropologique. Pour identifier des faits de communication linguistique, il faut considérer des échanges de signes qui se font dans le contexte d'une forme de vie semblable à la nôtre. Or ce qui caractérise une forme de vie comme humaine, c'est qu'on y retrouve des façons de faire établies, des coutumes, ou encore

\footnotetext{
1. Jerry Fodor et Ernest LePore, Holism : A Shopper's Guide, Oxford, Blackwell, 1992.
} 
des pratiques, bref des institutions, et que ce sont ces institutions qui déterminent le sens des actes ou des conduites. Pour que des pratiques puissent être reconnues par nous comme linguistiques, il est nécessaire que nous y retrouvions divers "jeux de langage" (questionner, ordonner, déclarer, etc.). Il doit y avoir, non pas bien entendu nos usages établis, mais des usages établis, et non seulement des usages établis par des individus à leur propre intention, mais des usages socialement établis, c'est-à-dire des attentes normatives définies préalablement à tout exercice particulier du langage entre tel et tel membres de la communauté linguistique.

L'argument de Fodor-LePore ne vise pas directement à contrarier cette version du holisme, mais une forme plus spéciale et (en apparence au moins) plus technique, qu'ils appellent le "holisme sémantique". Ce holisme se définit par son opposition à l'atomisme sémantique. Alors que l'atomisme pose que la relation sémantique fondamentale est celle qui va du signe à la chose du monde (ou bien de la chose du monde au signe dans l'esprit), le holisme sémantique soutient que les relations sémantiques fondamentales sont d'abord des relations entre les signes, à l'intérieur d'une totalité linguistique formée par ces signes ${ }^{2}$.

D'après nos auteurs, un holisme sémantique conséquent doit concéder que la communication humaine est un phénomène précaire. Si le sens est une propriété collective des signes (une propriété que chaque signe doit à son intégration à titre de partie dans une totalité linguistique ou dans un système), alors le sens d'une phrase dépend de la totalité à laquelle appartient cette phrase. Par conséquent, le sens d'une phrase prononcée ou écrite par un individu dépend du sens des autres phrases que cet individu a produites ou qu'il aurait pu produire. Quelque chose qui est matériellement la même phrase ne saurait avoir le même sens dans deux bouches différentes, à moins que toutes les autres phrases produites respectivement par ces deux locuteurs ne soient également identiques.

Les conséquences sont déroutantes. Par exemple, il y a parmi les phrases que nous pouvons produire des phrases que nos grands-pères n'auraient pas pu former (il suffit de fabriquer une phrase employant des termes introduits récemment dans la langue). Donc nous ne pouvons pas comprendre ce qu'ont écrit nos ancêtres, bien qu'à première vue ce soit du français et même souvent le même français que notre français. En effet, le sens dans lequel nous employons des mots tels que "bonjour" et "merci" est la propriété collective d'une collectivité de signes dont font aussi partie des signes d'usage récent tels que "je lui a envoyé un fax".

\footnotetext{
${ }^{2}$. Toute la question est de savoir ce qu'il faut entendre par une totalité des signes (j'y reviendrai dans la dernière section de ce texte).
} 
Soit maintenant le deuxième argument annoncé. Il résulte des analyses proposées par Dan Sperber dans l'ouvrage important qu'il a récemment publié sur les problèmes philosophiques fondamentaux d'une science anthropologique ${ }^{3}$.

En quoi consiste une communication, un échange verbal ? D'après Sperber, lorsque je comprends ce que me dit le passant dans la rue, cela veut dire en réalité que je me confectionne une version de ce qu'il a dit. Cette version que je produis est ma version, c'est ma représentation de la représentation (publique) qu'il m'a donnée, en s'exprimant, de son propos. La communication a lieu si ma version ressemble suffisamment à celle que mon interlocuteur a dans la tête. Elle ne saurait (et n'a d'ailleurs pas besoin) de lui être identique.

Autrement dit, pour entendre, non seulement les mots, mais le sens de mon interlocuteur, je dois payer de ma personne et lui attribuer (au style indirect) un discours (que je ne peux manquer de comprendre puisque j'en suis en réalité le véritable auteur). Revenons au petit échange initial. Lorsque je réponds "oui" à l'inconnu qui m'a demandé s'il y avait une boulangerie ouverte dans le quartier, je ne réponds pas tout à fait à mon interlocuteur. En effet, pour lui répondre, j'ai dû d'abord confectionner une version personnelle de ce qu'il me demandait. Ce passant m'a demandé (dans son langage) s'il y avait une boulangerie ouverte dans le quartier. Mais le discours rapporté (ci-dessus en italiques), justement, n'est plus l'œuvre d'autrui, il est produit par moi dans mon propre langage, et il est ensuite placé par moi, l'auteur, dans la bouche de mon interlocuteur à titre d'interprétation de ce qu'il m'a dit. Et c'est donc à ma version de sa question que je donne la réponse "oui". Or il n'y a jamais qu'une ressemblance (plus ou moins grande) entre les versions personnelles que les gens se font de ce qu'ils se disent.

Si vous me demandez : "Sommes-nous aujourd'hui le 23 mai 1997 ?", ma réponse sera "oui" tant qu'il s'agit de répondre à ma version de votre question. Quant à votre version de votre question, je n'y ait pas d'autre accès qu'"interprétatif". Par conséquent, si j'étais scrupuleux, je devrais vous répondre "à peu près oui", "c'est à peu près ça", autrement dit : oui, dans les limites de la ressemblance entre les idées (privées) que vous avez traduites dans votre discours extérieur et les idées (privées) par lesquelles j'ai moi-même interprété vos signes.

Je me propose dans ce qui suit de discuter ces positions de Sperber. Le netteté avec laquelle elles sont exposées dans La contagion des idées permet en effet de faire ressortir le présupposé philosophique qui sous-tend les conséquences énoncées. Dans son principe, la question que je voudrais poser est fort simple. Elle est la question qu'on a également envie de poser à Fodor et LePore. La voici : pourquoi ne pas dire que l'échange verbal donné en exemple est rendu trivialement possible par le fait de la communauté linguistique ? Par

\footnotetext{
${ }^{3}$. Dan Sperber, La contagion des idées - théorie naturaliste de la culture, Odile Jacob, 1996. (Le titre de ce livre sera désormais abrégé CI.)
} 
définition, si mon interlocuteur parle la même langue que moi, nous sommes capables d'avoir un échange de ce genre. Le monde dans lequel on peut envisager que toute communication soit précaire est un monde babélien : c'est un monde dans lequel des gens qui veulent communiquer ne le font pas en s'imposant d'user d'une langue commune, mais plutôt en s'efforçant de tirer parti de ressemblances qu'ils croient observer entre leurs idiolectes respectifs.

Il se trouve que la réponse invoquant une langue commune est exclue par ces philosophes. Fodor et LePore, dans leur livre, n'envisagent pas qu'un holiste veuille la donner (il est vrai que les théories holistes qu'ils critiquent ne font pas appel à quelque chose comme la langue). Quant à Sperber, il l'écarte explicitement. Pour lui, parler de langue commune serait du verbiage, car ce serait introduire une pseudo-entité.

Il n'est pas excessif de dire que c'est toute la philosophie des sciences sociales qui est ici en jeu. Sperber soutient, à juste titre, que le fond de la question est finalement à chercher sur le terrain ontologique.

\section{Questions de méthode et questions de réalité.}

L'argument de Sperber suppose que toute communication repose sur une interprétation : pour que je sache ce que me dit mon interlocuteur, il ne suffit pas que j'entende ce qu'il me dit (que je perçoive les signes qu'il m'adresse), il faut encore que je le fasse parler en construisant un discours (le plus souvent, en décidant que les signes qu'il m'adresse veulent dire ce qu'ils voudraient dire si c'était moi qui les avais utilisés à sa place). Ce présupposé a manifestement des conséquences pour les sciences sociales (conséquences qu'il développe lui-même dans son livre). L'une de ces conséquences est que ces sciences, si leur intention est de restituer le sens des actions, des coutumes et des croyances humaines, ont forcément un statut herméneutique. Entendre par là : le régime de la preuve dans ces disciplines n'est pas celui du renvoi à des faits (ce qui serait un régime descriptif). On ne peut pas prouver que telle signification est ou non donnée, que l'interlocuteur a telle ou telle idée. Tout ce qu'on peut prouver est qu'une manière de comprendre est plus recommandable qu'une autre à tel ou tel point de vue (qu'elle est plus orthodoxe, ou bien plus cohérente, ou bien plus plaisante). Il y a toujours plusieurs manières de lire les textes ou d'interpréter les données. S'il en est ainsi, cela veut dire que la fonction du lecteur est de faire parler les données ou les textes. Et c'est là ce qui justifie le qualificatif "herméneutique" pour caractériser son art. L'herméneutique trouve dans les textes des idées et des messages qui n'y sont pas sur le mode d'une présence factuelle, mais seulement sur le mode d'une présence interprétative.

Sperber formule d'ailleurs lui-même une thèse qui évoque le principe gadamérien de "l'universalité de l'herméneutique". Il note que, dans sa conception de la communication, 
l'interprétation commence avec le plus petit échange linguistique (il n'y a pas besoin de la limiter à des textes sacrés ou des paroles fondatrices).

Le processus de la communication se décompose en deux processus d'interprétation : l'un du mental vers le public, l'autre du public vers le mental (...) S'exprimer ou comprendre ce qu'autrui exprime, c'est déjà interpréter, de façon plus ou moins implicite. En outre, nous faisons tous un travail d'interprétation explicite lorsque nous répondons à des questions telles que : Qu'a-t-il dit ? Que penset-elle ? Que veulent-ils ? Pour répondre, nous représentons des contenus de propos, de pensées ou d'intentions au moyen d'énoncés de contenu semblable. (CI, p. 52)

Peut-on, dans ces conditions, étudier les représentations culturelles ${ }^{4}$ de façon objective ? C'est une des questions que pose Sperber. Cette question peut sembler méthodologique et, en un sens, elle l'est. Pourtant, la querelle de méthode ne peut pas être discutée sur le seul terrain de la méthodologie, car ce qui est en cause est plutôt l'ontologie des représentations culturelles. Voici comment Sperber explique le conflit en question.

Lorsque nous parlons des représentations, dit-il, nous usons d'un mot qui est en réalité appliqué à deux genres de choses : à des représentations publiques, qui existent matériellement dans l'environnement des personnes (comme les énoncés oraux ou écrits et tous les signes publics), ou bien à des représentations "mentales ou internes" (l'exemple donné est celui des souvenirs). La question est alors celle-ci : "Qu'est-ce qui est plus fondamental, les représentations publiques ou les représentations mentales ?" (CI, p. 108). La question n'est pas : qu'est-ce qui vient en premier dans l'ordre du connaître ? Qu'est-ce qui doit être connu en premier lieu, rendant possible ainsi la connaissance d'autre chose ? La question est bel et bien : qu'est-ce qui vient en premier dans l'ordre de l'être ? Qu'est-ce qu'il doit y avoir pour qu'il existe quelque chose comme ce que nous appelons des représentations ?

Sperber rappelle qu'il existe deux lignes de pensée à ce sujet. Je les opposerai en parlant pour la première d'une "théorie mentale" des signes ${ }^{5}$ et pour la seconde d'une "théorie anthropologique" des signes. La théorie mentale est celle qui dit : c'est l'esprit (ou le cerveau) du sujet qui possède (ou contient) les représentations. Le monde est représenté au sujet s'il est représenté dans son esprit (ou dans son cerveau, mais de toute façon dans une partie du sujet). L'autre théorie peut être qualifiée d'anthropologique dans la mesure où elle pose ceci : c'est la créature vivante qui a des représentations animales (de type perceptif), et c'est la créature humaine (l'animal qui a une forme humaine de vie) qui a des représentations

\footnotetext{
${ }^{4}$. Le mot de "représentation culturelle" est pris par Sperber dans un sens large qui inclut des discours (un conte), des cérémonies (la messe), des objets rituels (un masque), des règles (un article du code civil), des techniques.

${ }^{5}$. Dans le sens où la langue philosophique anglaise permet parler de "philosophie mentale" (mental philosophy).
} 
culturelles (symboliques ou linguistiques). Autrement dit, c'est l'homme tout entier (l'être capable d'un comportement humain), ce n'est pas une partie de l'homme (esprit ou cerveau).

\section{Les représentations}

Où est l'ontologie dans cette affaire ?

Nous sommes sur le terrain de l'ontologie parce que, même lorsque nous posons la question épistémologique de l'objectivité des connaissances obtenues dans le cadre d'une recherche anthropologique, nous le faisons en posant une question qui est la suivante : Que doit-il y avoir dans le monde pour qu'il y ait dans le monde quelque chose comme des représentations?

Il est vrai que la forme de cette question peut sembler inutilement contournée. Pourquoi ne pas résoudre le problème ontologique de la représentation par une réponse franche et nette qui dirait tout simplement : il y a dans le monde des représentations s'il y a dans le monde des entités qui soient des représentations (autrement dit, s'il y a dans le monde des entités identifiables qui soient identifiables comme des représentations) ?

La réponse est tentante, aussi bien pour le théoricien mentaliste que pour le théoricien sociologue. L'un et l'autre donneront des exemples. Le mentaliste pourra faire comme Sperber à propos des représentations privées (internes) et fournir un exemple d'événement cognitif : il y a dans le monde des représentations, pas exemple des souvenirs. De son côté, le partisan du holisme anthropologique pourra citer comme exemples de représentations publiques ceux que mentionne également Sperber. Il dira : il y a dans le monde des choses qui sont des représentations, par exemple des discours, des signes, des objets (comme les masques), etc.

Malheureusement, ces réponses directes ne résolvent nullement le problème posé. Il ne suffit pas de dire : "Vous demandez s'il y a dans le monde des représentations ? Eh bien, il y en a, et je peux d'ailleurs vous en donner des exemples !" Et cela vaut pour le mentaliste comme pour son adversaire. Car les exemples qui seront cités par une école risquent fort d'être récusés par l'autre école.

On a vu que le concept de représentation est communément appliqué à deux sortes d'entités : les représentations "internes" et les représentations publiques. Or il apparaît vite qu'elles ne peuvent pas être seulement deux sortes de représentations, c'est-à-dire deux espèces d'entités d'un même genre, comme si toutes les représentations étaient représentatives de ce qu'elles représentent au même titre. En réalité, le concept de représentation n'est pas le même selon qu'on donne la première place aux privées ou aux publiques. C'est pourquoi la réponse ontologique à donner suppose une analyse (ontologique) de ce qui doit être donné dans le monde pour qu'il y ait représentation.

Considérons d'abord ce que dit le mentaliste. Pour lui, il n'y a de représentations publiques qu'en un sens dérivé. Supposons qu'on lui dise : il y a dans le monde des 
représentations, par exemple cette inscription dans le marbre ou encore ce masque en bois. Il protestera qu'on ne peut pas identifier sans plus de telles entités comme des représentations. Comme objets physiques, ces choses existent de plein droit. Comme représentations, elles existent à condition de représenter quelque chose pour quelqu'un, donc à condition que les gens se représentent quelque chose à leur sujet. (Notons que, jusqu'ici, l'anthropologue holiste n'a rien à redire à un tel rappel de la définition du signe institué.)

Le mentaliste raisonnera de la façon suivante (cf. CI, p. 108) : les représentations privées peuvent exister sans une contrepartie publique (puisque je peux avoir une pensée sans l'exprimer); en revanche, les représentations publiques ne peuvent pas exister sans une contrepartie privée (sinon elles ne représentent pas quelque chose pour quelqu'un). Par conséquent, vous ne pouvez pas établir qu'il y a des représentations publiques rien qu'en montrant du doigt des signes en train de circuler, les livres, les signaux publics, les sonneries du téléphone, le bruissement des conversations. En effet, il vous sera répondu que toutes ces choses ne sont pas des représentations par elles-mêmes.

Les représentations publiques sont liées à ce qu'elles représentent seulement à travers la signification que leur attribuent leurs producteurs ou leurs utilisateurs; elles n'ont pas de propriétés sémantiques propres. En d'autres termes, les représentations publiques n'ont de signification qu'à travers leur association avec des représentations mentales. (CI, p. 112)

Par conséquent, conclut le mentaliste, il ne peut y avoir dans le monde des choses qui soient des représentations publiques que s'il y a dans le monde autre chose pour leur conférer cette réalité. Cette "autre chose" doit remplir une condition : être capable de conférer la signification aux représentations (donc avoir des "propriétés sémantiques propres").

Les seules véritables représentations sont donc les représentations "internes" (celles qui sont intrinsèquement représentatives). Il n'y a pas vraiment de représentations publiques, mais il y a des choses publiques que les gens se représentent, en privé, comme étant des représentations publiques. Ils peuvent comprendre les représentations publiques parce qu'ils ont dans l'esprit (dans la tête) des représentations privées grâce auxquelles ils transfèrent du sens aux objets publics destinés à le recevoir.

Comme on sait, ces considérations ne paraissent décisives qu'à ceux qui ont déjà adopté la conception mentaliste. Le partisan de la théorie anthropologique leur oppose un raisonnement qui, lui semble-t-il, suffit à les vider de leur force. Dans son exposé sur ce point, Sperber n'a pas rappelé ce raisonnement, mais on peut le faire facilement.

De nouveau, la question posée est : Y a-t-il dans ce monde quelque chose qui soit les représentations dont nous parle le théoricien de la culture ? Nous connaissons la réponse du mentaliste : Oui, il y a des choses de ce genre. Par exemple, il y a les souvenirs. 
Mais ici, le partisan d'une conception anthropologique des signes fera une objection en deux temps.

$1^{\circ}$ Première étape : critique de la notion de signes intrinsèquement signifiants. D'abord, il demandera qu'on lui précise en quel sens il faut entendre les "représentations internes". Nous savons, dira-t-il, ce que c'est qu'une chose publique faisant office de mémorial (par exemple une statue ou une plaque commémorative). Nous pouvons comprendre également ce que c'est qu'un mémorial à usage privé (des notes dans son carnet personnel, un nœud au mouchoir). Publics ou privés, ces signes mémoriaux ont une signification parce qu'ils sont utilisés par quelqu'un pour se remettre en mémoire quelque chose. Mais le théoricien mentaliste nous invite à introduire maintenant des signes intrinsèquement mémoriaux. Or nous ne voyons pas ce que pourraient être des choses présentes au sujet (présentes d'une présence "mentale") qui seraient intrinsèquement des mémoriaux (internes), c'est-à-dire des choses présentes (dans l'esprit) représentant au sujet le passé. On comprend bien pourquoi un mémorial en marbre n'est pas intrinsèquement représentatif du passé. Mais pourquoi un mémorial "interne" le serait-il ? Le sujet du souvenir ne peut pas être renvoyé au passé par le simple fait d'avoir en face de lui une stèle commémorative : il faut qu'il se serve de cette stèle comme d'un mémorial. Mais comment sera-t-il renvoyé au passé par une donnée mentale présente, alors qu'il n'est pas renvoyé au passé par une donnée extérieure présente?

La notion qui fait difficulté n'est pas celle de souvenir, si l'on entend par "avoir un souvenir" tout simplement "se représenter un épisode du passé". Mais "se représenter un épisode du passé" n'équivaut nullement à avoir dans la tête une chose qui soit une représentation du passé, donc à se représenter que quelque chose (de présent) est une trace du passé, et encore moins se représenter qu'une chose présente est un signe destiné à tourner notre esprit vers le passé à la façon d'une stèle commémorative. La notion qui fait difficulté est celle d'une chose qui représente le passé au sujet sans que ce sujet ait à se servir de l'indice présent pour évoquer le passé. Le mentaliste a raison de dire qu'un signe mémorial public ne représente rien par lui-même à quelqu'un qui n'est pas capable de s'en servir pour se souvenir du passé, mais le même raisonnement s'applique à des signes mémoriaux "mentaux" ou "internes".

De façon générale, s'il y a des signes mentaux purement internes, comment fait-on pour les interpréter ? Il y a bien une condition côté sujet pour qu'un signe commémoratif soit donné comme signe au sujet : mais cette condition n'est pas la présence dans sa tête d'une "contrepartie privée", c'est-à-dire d'un signe commémoratif interne, c'est la possession par le sujet d'une capacité à se servir de signes (publics ou personnels) comme de signes du passé.

$2^{\circ}$ Deuxième étape : nécessité d'une contrepartie publique pour les représentations privées. (On entend ici par "représentations privées" celles que le sujet "garde pour lui"). Le partisan de la théorie anthropologique des signes retournera maintenant au mentaliste l'objection que faisait ce dernier, et il lui dira ceci : il n'y a des signes dans ma tête (par 
exemple des phrases que je construis en silence à mon propre usage) que si nous pouvons leur trouver une "contrepartie publique". Le mentaliste disait tout à l'heure : le signe public (panneau de signalisation ou masque nègre) ne signifie pas par lui-même, il signifie quelque chose pour quelqu'un, donc à condition que quelqu'un soit là pour se représenter quelque chose par le moyen de ce signe. Il faut donc, c'était la conclusion mentaliste, une contrepartie privée. Mais nous demanderons maintenant : que faut-il se représenter ? Celui qui connait le code de la route comprend le sens du signal routier, celui qui a été initié au culte des masques comprend la valeur et la fonction du masque dans la cérémonie, ceux qui n'ont pas appris le code de la route ou la voie des masques n'y comprennent rien ou en sont réduits à des hypothèses, à des "interprétations" (ce qui trahit le fait qu'ils ne comprennent pas). Pour qu'il y ait, non pas seulement une représentation dans la tête de l'utilisateur du signe, mais bien la représentation correcte, il faut que l'utilisateur saisisse correctement ce que lui dit le signe. Ce n'est pas lui qui décide. Par conséquent, pour qu'il y ait la représentation correcte de ce que communique le panneau de passage interdit, ou encore une représentation correcte du statut qu'indique le masque, il faut que la représentation dite "interne" (personnelle) ait une contrepartie publique, à savoir l'institution d'une pratique dans laquelle l'usage de ces signes a été établi et (éventuellement) codifié .

La controverse sur la notion de signe ou de représentation tourne donc au dialogue de sourds. Il se découvre que les deux écoles paraissent seulement partager un vocabulaire pour présenter l'objet de leur différend, mais qu'elles ne sont pas en mesure de s'entendre sur une première délimitation de cet objet. Elles ne parviennent pas à s'accorder sur la position même de cet objet : voici quelque chose qui est un signe. Pour le mentaliste, un mot écrit sur une porte ("SORTIE") ou une carte routière ne sont pas véritablement des signes. En réalité, dirat-il, ils ne le sont pas du tout, mais peuvent le devenir grâce à d'autres réalités qui, elles, sont par elles-mêmes des signes ou des représentations. Ils n'ont pas de "propriétés sémantiques propres". Pour l'adversaire holiste de ce mentaliste, les "signes mentaux" et les "représentations internes" dont parle la théorie mentaliste usurpent cette appellation, car ils ne ressemblent en rien à ce qu'on appellerait normalement un signe ou une représentation. Par exemple, selon le mentaliste, il y a des "images", voire des "cartes mentales" dans l'esprit du sujet, mais il ne s'agit nullement d'images que le sujet doive examiner pour voir ce qu'elles représentent ni de cartes qu'il doive consulter pour déterminer son itinéraire. Autrement dit, le holiste déclare ne pas comprendre ce que c'est qu'une chose naturelle dotée de propriétés sémantiques intrinsèques.

Puisque le désaccord porte sur les notions les plus élémentaires d'une théorie des signes, ce n'est pas à cette théorie (la sémiotique ou la sémantique) qu'on peut demander de faire avancer la discussion. Nous sommes obligés de reprendre la question de plus loin. Je crois, d'accord sur ce point avec Sperber, qu'il faut s'attacher d'abord à éclaircir ces notions élémentaires sur le terrain de l'ontologie. 
Le problème n'est pas de choisir entre le positivisme et l'herméneutique (faut-il s'en tenir aux faits ou doit-on faire parler les données ?). Il est de choisir entre le naturalisme et l'intentionalisme $^{6}$. En disant cela, je me range bien volontiers du côté de Sperber en ce qui concerne l'un des points du débat qu'il a avec Clifford Geertz.

La question que doit se poser l'anthropologue, écrit Geertz, n'est pas celle du statut ontologique des objets dont il traite (fêtes, usages, mythes, etc.) mais celle de leur signification. La discipline n'aurait pas, selon lui, à se prononcer sur le statut ontologique des objets qu'elle veut étudier, car ce statut est connu : le combat de coqs balinais ou la razzia marocaine (allusion à deux célèbres analyses qu'on doit à Geertz) sont "des choses de ce monde", comme les pierres et les rêves. En revanche, le problème à poser est celui de "ce qui est dit quand ces événements se produisent". Sperber cite ce passage de Geertz :

La question à poser à propos d'un clin d'œil délibérément exagéré ou d'une razzia sur des moutons servant de démonstration de force [deux des exemples que Geertz donne de représentations publiques] n'est pas de savoir quel est leur statut ontologique. Ce statut est le même que celui des pierres ou celui des rêves - ce sont des choses de ce monde. La question est de savoir quelle est leur portée : qu'est-ce qui est dit à travers de tels événements lorsqu'ils se produisent ? (The Interpretation of Cultures, cité dans CI, p. 110; la parenthèse est ajoutée par Sperber.)

Il va de soi que la question du sens des événements doit être posée : on attend justement de l'ethnologue qu'il nous restitue le sens de ces pratiques pour nous énigmatiques. Mais cela veut-il dire qu'on n'ait pas à s'entendre préalablement sur le statut ontologique de ce qu'on va ensuite analyser du point de vue de la signification ? Sperber fait une objection à la trivialisation de la question ontologique qui me paraît pleinement justifiée.

Pour ma part, la raison qui doit être invoquée ici est d'abord celle-ci : donner à toutes les choses réelles le statut de "chose de ce monde", c'est leur donner le même statut. La notion de statut devient donc inutile. Pourtant, il est remarquable qu'on parle assez communément de "statut ontologique", ce qui pourrait bien indiquer qu'on a du mal à ne pas introduire une certaine dose de diversité dans sa vision ontologique des choses. En effet, la notion d'un statut qui est ici pertinente est celle d'une position (celle d'une personne ou d'une chose) à l'égard d'autres positions du point de vue d'un système (par exemple, d'un système juridique).

\footnotetext{
${ }^{6}$. Par "intentionalisme", on peut entendre une doctrine portant sur les attributions intentionnelles. On appellera "attributions intentionnelles" des propositions du genre "il croit que le train va arriver" ou "il prépare le dîner" (= il fait différentes choses dans l'idée qu'il accomplit par là les opérations nécessaires à la préparation d'un dîner). Ces propositions attribuent à des gens des idées et des intentions. La doctrine intentionaliste est que ces propositions sont d'honnêtes propositions descriptives, autrement dit qu'elles ont une valeur de vérité. Ces propositions ne sont pas comparables à des grimoires réclamant de la part d'un lecteur une projection herméneutique.
} 
L'intérêt de la notion de "statut" était donc de nous inviter à concevoir des positions respectives : de même que l'héritier est l'héritier d'un défunt sous le rapport d'une succession, de même la catégorie de changement, par exemple, va se comprendre relativement à celle de l'état d'une chose, ou la catégorie de compétence relativement à celle de performance. Ce caractère positionnel du statut est perdu dans la conception égalitaire que reprend Geertz : toutes les choses du monde ont le même statut (elles sont toutes également "de ce monde").

Sperber a donc raison, je crois, de commenter ainsi le texte de Geertz qui vient d'être cité :

L'ontologie n'a pas pour tâche de dire quelles choses sont "de ce monde" et quelles choses n'en sont pas. Elle a pour tâche de dire de quelle manière (au singulier ou au pluriel) les choses peuvent être de ce monde. Or pour ce qui est des choses culturelles, le problème reste entier. (CI, p. 111)

Poser la question ontologique à propos d'un genre de choses, c'est en effet demander comment les choses dont on parle s'inscrivent dans le monde, et ce peut être, le cas échéant, de savoir comment elles s'inscrivent "matériellement" ou "phénoménalement" dans le monde. Ayant ainsi marqué mon accord avec Sperber sur ce premier point, je vais maintenant me tourner vers la question posée par la précision qui figure dans la parenthèse : y a-t-il une ou plusieurs manières pour une chose de faire partie du monde ? Ontologie moniste ou ontologie pluraliste ? Sur ce second point, il faudra malheureusement prendre congé de Sperber, car je ne pense pas qu'il ait bien exposé ce qui distingue un pluralisme d'un monisme ontologique.

\section{Le pluralisme ontologique selon Sperber}

Si Sperber donne la primauté aux représentations internes sur les représentations externes (publiques), c'est parce qu'il estime que les théories cognitivistes contemporaines nous permettent de concevoir qu'un objet mental soit aussi un objet matériel. En revanche, l'identification matérielle des signes publics est selon lui problématique. Certes, une représentation publique correspond bien à un objet matériel, par exemple le masque rituel à un morceau de bois, la parole prononcée à une suite de sons. Mais il reste à expliquer, estimet-il, comment ces objets matériels ont une signification publique.

\footnotetext{
"Quand on passe aux représentations culturelles, prétendument dotées de significations publiques, la situation est bien différente [que pour les représentations mentales telles que les souvenirs ou les raisonnements] (...) La vérité est que nous n'avons aucune idée de la manière dont ces représentations culturelles pourraient être "des choses de ce monde" " (CI, p. 111; les mots entre guillemets font allusion à l'expression de Geertz citée plus haut).
} 
Le raisonnement de Sperber (que je qualifierai de mentaliste) est un raisonnement qui suppose que nous devions inscrire la chose culturelle dans le monde sous les traits ontologiques d'un objet. Un tel raisonnement, semble-t-il, est strictement parallèle à un raisonnement qu'on pourrait qualifier de "platoniste".

Voici les quatre étapes du raisonnement platoniste :

- (1) Il y a des représentations publiques.

- (2) Soit R l'une de ces représentations, par exemple le Conte du Chat botté ${ }^{7}$.

- (3) Aucun objet matériel n'est cette représentation R.

- (4) Donc il faut bien poser un objet immatériel qui soit cette représentation R.

Sperber refuse ce qu'il appelle le "pluralisme ontologique", c'est-à-dire qu'il refuse cette inférence par laquelle des objets immatériels sont ajoutés aux objets matériels. Comment de telles choses immatérielles pourraient-elles s'inscrire dans le monde ?, demandet-il fort justement.

Or le raisonnement mentaliste (que Sperber nous soumet) paraît être exactement parallèle à celui du "platoniste" (qu'il conteste), sauf qu'on s'imagine éviter les objets idéaux du "platonisme" en cherchant les réalités en question dans la sphère mentale. A l'étape $n^{\circ} 4$, on trouve maintenant ceci :

- (4 bis) Donc il faut bien poser un objet mental qui soit cette représentation R.

Quant au raisonnement qui est tout à la fois mentaliste et matérialiste, il se borne à ajouter que l'objet mental est doté d'une identité matérielle à l'intérieur du cerveau. C'est seulement au dehors qu'on n'a pas pu mettre le doigt sur un objet physique qui soit le Conte du Chat botté. En principe, on doit pouvoir le trouver au dedans. Il est vrai qu'on n'y trouvera pas le Conte (objet idéal), mais une version particulière du conte (objet empirique). Pour un mentaliste, les objets mentaux sont dans les têtes. Si le Conte du Chat botté est dans le monde en étant dans les têtes des gens, il y aura, non pas un seul et même conte, mais plusieurs contes semblables, plusieurs versions du Conte (autant que de têtes). On en arrive ainsi à l'idée maîtresse du livre de Sperber, qui est de faire coexister dans le même monde deux populations : la population des créatures humaines et la population des idées.

La conclusion du raisonnement mentaliste impose donc de réviser la proposition initiale : il n'y a pas, à proprement parler, des représentations publiques, mais il y a des classes ou des familles de représentations mentales (privées) qu'il est possible de traiter comme si elles formaient de telles représentations publiques.

En décidant de traiter les idées qui composent une culture comme une population d'entités matérielles (objets mentaux), Sperber entend sans doute préparer la constitution d'une science des cultures comme science de la diffusion des idées, donc de leur distribution

\footnotetext{
${ }^{7}$. A cette étape, nous estimons pouvoir donner un nom propre à une "représentation publique" (ici, un conte de fées). Nous conférons donc à cette représentation le "statut logique" d'un objet de référence identifiable, et il reste à lui trouver le "statut ontologique" d'une chose qui trouve sa place dans ce monde.
} 
entre les cerveaux. L'anthropologie pourra se modeler sur l'épidémiologie. Mais ce qui m'importe ici n'est pas de discuter ce modèle, c'est la justification ontologique qui en est donnée. Les êtres humains et les idées entreraient dans le même genre ontologique : dans un cas comme dans l'autre, nous aurions des entités individuelles, et même des entités d'ellesmêmes individuées (des objets). Dans un cas comme dans l'autre, on pourrait procéder à des recensements.

Par conséquent, le seul pluralisme des manières d'être une chose de ce monde que Sperber ait envisagé est celui qui distingue deux modes d'existence : le mode matériel de l'existence propre aux objets physiques et le mode immatériel de l'existence propre aux objets idéaux (ou "abstraits"). Ce sont bien là deux modes d'existence (pluralisme), mais deux modes d'existence pour des objets. Ce pluralisme est donc subordonné à un monisme ontologique selon lequel il n'est d'autre genre d'entités que celui des objets. Et c'est justement un tel monisme ontologique qui rend compte du parallèle remarquable entre le raisonnement mentaliste et le raisonnement platoniste.

\section{Critique du monisme ontologique}

Il me semble que, dans le raisonnement mentaliste qui nous occupe, la décision ontologique importante n'est pas celle qui pose des choses immatérielles (étape $n^{\circ} 4$ ), mais que c'est plutôt l'hypostase par laquelle la représentation devient un objet individuel susceptible de recevoir un nom propre (étape $n^{\circ} 2$ ).

Derrière cette hypostase, nous trouvons en effet un présupposé quant à l'ontologie. Il est présupposé que le statut ontologique de tout ce qui sera posé dans le monde à un titre ou à un autre sera le statut d'un objet (ou d'une famille d'objets). Le présupposé de l'étape $n^{\circ} 2$ du raisonnement ci-dessus est donc :

— (2 bis) : "il y a une représentation $\mathrm{R} "=$ "il y a un objet $x$ tel que $x$ soit la représentation R". (Peu importe à ce stade si l'on tient à préciser que cet objet est matériel ou si l'on accepte des "objets abstraits".)

En présence d'un tel présupposé, nous voudrions objecter ceci : s'il y a un conte qui est le conte du Chat botté, cela ne veut pas dire qu'il y a dans le monde un objet qui est ce conte, mais qu'il y a dans la littérature des contes de fées un tel conte. Nous devons donc nous tourner vers le mode de présence mondaine des formes et des œuvres littéraires. Le mode d'inscription du conte du Chat botté dans le monde, son mode de présence effective consistera à être raconté, transmis, consigné, réécrit, etc. Comment la littérature est-elle présente dans le monde ? Pour trouver le conte en question, nous ne cherchons pas parmi des objets, mais plutôt parmi des actes. Voici un conteur en train de parler : son acte est-il la récitation d'un conte ? Si oui, est-ce la récitation d'un conte ou l'invention d'un conte ? Si c'est une récitation, est-ce celle du Chat botté ou celle de Cendrillon ? La question "ce conte 
existe-t-il ?" ne nous conduit pas à chercher sa réalité parmi les objets matériels "extérieurs", ni, faute de l'y trouver, parmi les objets immatériels ou encore parmi les objets matériels intérieurs à l'organisme, mais dans une autre catégorie que celle des objets.

Mais ici, nous nous heurtons à une profonde divergence des philosophes, non pas tant sur telle ou telle thèse ontologique que sur l'idée de ce que nous demandons à un éclaircissement ontologique. En effet, l'idée même d'une différence ontologique, c'est-à-dire d'une différence dans la manière d'être - ou différence entre des catégories - est loin de faire l'unanimité chez les philosophes (peut-être en raison de l'obscurité de bien des exposés consacrés aux "catégories").

Pour introduire cette idée d'une différence dans le mode d'être des "entités", je partirai pour ma part de la notion communément reçue de statut ontologique. Si la notion a un intérêt, elle doit vouloir dire ceci : toute chose qui a un statut ontologique "existe" ou "est une chose de ce monde", mais pas au même titre. Ici, c'est exister, c'est exister à tel ou tel titre ${ }^{8}$ (comme l'on dit : être intéressé à telle affaire "à tel titre", par exemple en tant qu'héritier, que donateur, etc.). Il faut donc préciser la "catégorie" de l'existence ou de l'individuation.

En fin de compte, c'est la notion de "genre d'objets" qui demande à être éclaircie si l'on veut définir l'opposition du "monisme" et du "pluralisme". Wittgenstein a bien illustré la nécessité pour le philosophe de préciser sa pensée sur ce point ${ }^{9}$. L'occasion de sa distinction lui est fournie par les philosophes qui, voulant distinguer les choses physiques et les les données sensibles (par exemple visuelles), en viennent à dire que l'objet physique et la donnée des sens (par exemple l'arbre physique de la nature et l'arbre en tant que donnée de la perception) sont deux genres d'objets ${ }^{10}$. Wittgenstein nous demande de considérer le contraste des deux propositions suivantes :

(1) Un train de chemin de fer, une gare de chemin de fer et un wagon de chemin de fer sont des genres d'objets différents (A railway train, a railway station and a railway car are different kinds of objects).

(2) Un train de chemin de fer, un accident de chemin de fer et un règlement de chemin de fer sont des genres d'objets différents (A railway train, a railway accident and a railway law are different kinds of objects).

\footnotetext{
${ }^{8}$. En termes plus traditionnels, on dira que ce qui existe existe toujours sous une forme ou une autre.

9. The Blue Book, Oxford, Blackwell, 1958, p. 64. La clarification demandée ne passera pas forcément par une reprise de la doctrine traditionnelle des catégories. D'ailleurs Wittgenstein n'emploie nullement le mot "catégorie", mais se contente de s'intéresser à l'expression "a different kind of object". Ce qui importe ici n'est pas de retrouver le vocabulaire classique, mais de comprendre la raison d'être d'une différence entre des types d'entités, différence irréductible à une simple différence générique.

${ }^{10}$. Quelqu'un qui tiendrait la question du statut ontologique pour triviale pourrait dire : l'arbre naturel et l'arbre visible sont, tout comme la pierre et le rêve, des "choses de ce monde"; ce sont seulement différents genres d'objets.
} 
Nos philosophes croient procéder à des distinctions entre genres d'objet telles que celles offertes par la première de ces propositions : ces différences donnent lieu à une classification, à une taxinomie. Pourtant, selon Wittgenstein, la distinction qu'ils veulent faire est plutôt du type (2). C'est une différence dans le mode d'existence, dans le genre ontologique ${ }^{11}$.

Leur erreur, explique Wittgenstein, porte sur la grammaire du mot "genre" (the grammar of the word "kind"). Erreur comparable à celle des philosophes qui disent qu'un nombre est une sorte d'objet qu'un chiffre (et cela pour réagir contre l'autre école, formaliste, laquelle voudrait que les opérations que nous faisons apparemment sur les nombres portent en réalité sur des objets concrets, tels que des jetons ou des inscriptions). Dans cette alternative — ou bien le nombre se confond avec un objet empirique (le chiffre), ou bien il doit en être distingué et posé à titre d'objet idéal —, nous avons l'analogue de l'alternative entre un raisonnement mentaliste et un raisonnement platoniste évoquée ci-dessus.

Monisme et pluralisme se servent d'images différentes pour se donner la notion d'une diversité ontologique. Le moniste use de l'image d'un inventaire notarial. Il parle, selon la formule familière aux philosophes britanniques, d'un "ameublement du monde" ${ }^{12}$. Le pluraliste trouve plutôt ses images de diversité dans des systèmes, des totalités offrant une complexité. Ainsi, chez Wittgenstein, le modèle est grammatical : les différences de catégories se présentent comme les différences qu'on fait entre les "parties du discours".

On fait l'inventaire de "l'ameublement du monde", sinon en répertoriant toutes les pièces du mobilier (une à une), du moins en fixant les grands genres : tables, fauteuils, canapés, etc. L'exemple qu'a pris Wittgenstein suggère ici une image. Un recensement des "genres d'objets" conçu sur le modèle d'un inventaire des objets par "genres" peut donner lieu à une liste unique. Cette liste sera celle des objets du monde, chacun d'eux étant conçu comme une "entité", une unité de compte. L'ontologie moniste conçoit les entités comme des articles identifiables, au sens commercial du mot "article", donc au sens d'une unité figurant dans l'inventaire du fonds de commerce ${ }^{13}$.

\footnotetext{
${ }^{11}$. Le mot "catégorie" ne suffit pas à marquer si la différence visée est de type (1) ou de type (2), car il est aujourd'hui pris souvent dans le sens taxinomique. Impossible de se dispenser d'un éclaircissement philosophique sur la portée des différences ontologiques.

${ }^{12}$. Sperber appartient lui-même à cette tradition quand il écrit que les termes généraux dont se sert l'anthropologue n'ont pas de valeur descriptive (n'ont pas de portée ontologique), car "l'usage approprié de termes tels que "mariage", "sacrifice” ou "chefferie" ne nous dit pas si les mariages, les sacrifices ou les chefferies font partie des choses qui meublent le monde" (CI, p. 31).

${ }^{13}$. En anglais, les philosophes empruntent justement au vocabulaire des énumérations et des listes le mot "item" pour signifier une existence distincte devant donner lieu à une mention distincte dans la liste donnant l'inventaire de l'"ameublement du monde".
} 
Supposons que nous achetions les éléments d'un jeu de train électrique. Le jeu se présente comme un ensemble de pièces démontées qu'il faut assembler. La notice fournie par le producteur doit annoncer combien de pièces distinctes on va trouver dans la boîte. On aura donc 250 pièces, à savoir tant de rails, tant d'aiguillages, tant de wagons, tant de gares, etc. Nous pouvons demander : combien de wagons, combien de gares, combien de locomotives, etc. ? Dans ce cas, nous appliquons un principe d'individuation permettant de distinguer un exemplaire d'un autre exemplaire dans le même type. Mais nous pouvons aussi réunir tous ces objets dans une seule liste, et cela illustre le fait qu'il n'y a dans cette perspective qu'un genre ontologique (qu'un seul mode d'existence prévu).

Dans cette optique, le philosophe qui professe une ontologie moniste pourrait dire : je consentirai à admettre qu'il y a dans le monde des accidents de chemin de fer et des règlements de chemin de fer le jour où l'on aura identifié des objets matériels qui soient ces accidents ou ces règlements. Ne me demandez pas d'admettre autre chose, car je me refuse à poser des entités platoniques.

Considérons maintenant une classification de type (2). Il n'y a pas de liste concevable dans laquelle on pourrait inclure des articles variés tels que, d'une part, les diverses pièces matérielles et, d'autre part, les diverses dispositions du règlement. L'ontologie ne se fera pas dans l'optique d'établir (en principe) une liste unique de tous les articles du système, mais plutôt d'arriver à une table synoptique de listes. La question ontologique (par exemple, y a-t-il quelque chose comme des règles dans le monde du chemin de fer ?) est donc légitime, compréhensible, mais sous la condition de se placer à chaque fois dans une catégorie. Pas de recensement concevable de l'"ameublement", du "mobilier" ontologique de l'univers.

Il n'est pas indifférent que l'exemple pris par Wittgenstein soit celui de mots formés à partir d'une référence au chemin de fer. En français, on pourrait prendre pour exemple les "entités ferroviaires" : le matériel roulant ferroviaire, les règlements ferroviaires, etc. On trouve bien des relations entre tout cela (de même, pour reprendre l'exemple d'Aristote, qu'entre toutes les choses qui sont dites "saines"). Certaines de ces relations ont le caractère systématique de relations internes : s'il n'y avait pas du matériel ferroviaire et des déplacements ferroviaires, il ne saurait y avoir des accidents ferroviaires. L'existence des accidents de la circulation ferroviaire suppose qu'il y ait des trains qui circulent : la catégorie à laquelle appartiennent ces événements dépend ontologiquement de la catégorie des objets à laquelle appartiennent les locomotives et les passages à niveaux ${ }^{14}$.

Mais ces relations ne renvoient nullement à une signification générique susceptible de servir à l'établissement d'une liste générale de toutes les choses qui sont ferroviaires.

\section{L'élimination métaphysique des représentations communes}

\footnotetext{
${ }^{14}$. La dépendance est ontologique ou métaphysique, pas physique.
} 
Comment se pose la question des représentations culturelles dans la perspective d'une ontologie moniste ? Le principe fondamental est qu'il ne saurait y avoir quelque chose (qu'il s'agisse d'une personne, d'un conte ou d'un code juridique) que s'il existe un objet identifiable comme étant ce quelque chose. Dans ces conditions, les candidats à l'existence paraissent être de deux ordres : certains sont des objets "concrets" (matériels, mondains), d'autres sont des objets "abstraits" (non localisés, idéaux). Par exemple, le Conte du Chat botté, s'il existait une entité qui soit (par elle-même) cette représentation publique, ne pourrait être qu'un objet abstrait. En effet, mon exemplaire imprimé du Conte est un objet "concret" (qui prend de la place sur le rayon de ma bibliothèque), mais pas le Conte lui-même.

Est-ce par un parti pris matérialiste ou par un parti pris nominaliste ? Quoi qu'il en soit, Sperber juge que les objets abstraits dont nous parlent certaines théories sémantiques sont indésirables. Il va donc procéder à leur "élimination" par réduction, en usant ici de la procédure nominaliste familière d'élimination des hypostases "platoniciennes". Il n'y a pas l'Homme en soi ou le Lit en soi (objets abstraits), il y a des hommes en chair et en os ou des lits en bois, c'est-à-dire des individus matériels et empiriques qui se ressemblent suffisamment pour qu'il soit possible d'hypostasier leur ressemblance. Il s'agit maintenant de transposer cette technique de réduction aux "représentations communes" considérées comme des objets platoniciens (le Conte du Chat botté, la Langue française). On montrera que ces objets "abstraits" n'ont par eux-mêmes aucune réalité, que ce sont des constructions logiques qu'on se donne pour tenir lieu des objets concrets, un peu comme le comportement d'un unique "Français moyen" sert à représenter les comportements variés des divers Français.

Au point de départ, nous devons donc poser qu'il y a des ressemblances entre les représentations mentales que possèdent les individus. Qu'est-ce qui permet de le dire ? C'est le fait de la communication.

\footnotetext{
"En général les producteurs et les utilisateurs de représentations publiques leur attribuent des significations semblables, sans quoi ces représentations ne pourraient jamais servir à communiquer". (CI, p.112)
}

Sperber insiste sur le point suivant : il convient ici de parler de ressemblance et non pas d'identité. Pourquoi ? Les représentations se diffusent, non par réplication, mais par un travail d'interprétation (en raison des allers et retours entre la sphère privée et la sphère publique). Il écrit donc : "La communication humaine aboutit généralement à un certain degré de ressemblance, et non pas à une identité, entre les pensées du communicateur et celles de son destinataire" (CI, p. 115). On ne saurait aller plus loin et demander que ces représentations soient identiques. Nous retrouvons ainsi les raisons qui conduisent à penser que toute communication humaine est précaire. Ces raisons se tirent directement de l'ontologie présupposée par la théorie sperberienne du langage. 
Sur la base de ces ressemblances entre les représentations, il est possible de procéder à l'hypostase des représentations communes. Puisque les représentations mentales des individus - les "représentations concrètes individuelles" (CI, p. 115) — sont assez semblables pour être dites "partagées" par différentes personnes (chacun a sa version, mais les versions sont apparentées), on peut procéder à une opération que Sperber appelle "abstraction" et qui consiste, pour l'essentiel, à laisser tomber les différences entre individus ${ }^{15}$, ce qui a pour effet de substituer à la pluralité des individus une construction logique qui les représente (mais seulement dans leurs ressemblances).

\footnotetext{
"Ces similarités interindividuelles rendent possible de faire abstraction des différences individuelles et de décrire "la langue" ou "la culture" d'une communauté, "la signification" d'une représentation publique, ou encore de parler, par exemple de "la croyance" selon laquelle les sorcières voyagent sur des balais comme si l'on avait affaire à une unique représentation, indépendante de ses expressions publiques ou de ses réalisations mentales. Ce que l'on décrit est alors une abstraction." (CI, p. 112)
}

Dans ce texte, l'exemple d'une représentation publique qui nous est donné est celui d'une croyance traditionnelle à propos des sorcières. Cet exemple montre bien que ce qui importe à Sperber est la transition de plusieurs entités réelles (similaires) à une unique entité (irréelle). Pour se donner cette unique entité, il faut la produire par "abstraction" (comme on produit le portrait du "Français moyen" à partir des individus en chair et en os).

Sperber conclut de là qu'une abstraction ne saurait être une chose de ce monde (elle en est empêchée par son statut de construction logique). Il estime avoir par là réglé leur sort aux "représentations communes" et aux "significations publiques" des partisans du holisme culturel. Par exemple, on ne comptera plus les langues parmi les "choses de ce monde". Il n'y a pas la langue française, il y a une foule de discours qui présentent suffisamment de ressemblances pour qu'on puisse dériver l'hypostase de la langue.

Je ferai deux objections. La première porte sur l'ontologie des idées. La seconde porte sur l'ontologie des langues

\section{Ontologie des idées}

Aux yeux de son partisan, l'intérêt de l'ontologie moniste des idées est de procurer une notion suffisamment claire de ce que sont dans le monde les idées pour qu'on puisse s'engager dans l'élaboration d'hypothèses au sujet de leur histoire et de leur destin qui aient une chance

\footnotetext{
${ }^{15}$. Ici, les individus sont les idées (membres de la population des objets mentaux) que se font les individus humains de leur milieu et de leurs interactions.
} 
de pouvoir être appliquées, éprouvées, contrôlées par des preuves factuelles. Un programme de recherches peut être esquissé.

Encore faut-il que cette notion de ce que sont les idées (de leur ontologie) soit claire. Il me semble qu'elle ne l'est qu'en apparence.

Qu'est-ce que c'est en effet qu'une idée ? La psychologie cognitive qu'adopte Sperber est une psychologie contemporaine, c'est-à-dire qu'elle retient la grande leçon de la philosophie analytique de l'esprit. Lorsque Sperber dit qu'une idée est représentative, il n'explique pas cette représentativité de l'idée comme l'aurait fait un disciple de Descartes, de Locke ou de Mill, en la comparant à une image ou à un tableau. Il compare l'idée à une phrase. Autrement dit, il nous invite à analyser les actes mentaux, et aussi les états mentaux tels que la croyance, comme des "attitudes propositionnelles". Les idées ont cessé d'être des signes iconiques, elles sont devenues des signes propositionnels. Se représenter sur le mode de la croyance qu'il fait beau, ce n'est pas avoir à l'esprit une vive image de beau temps, c'est se rapporter sur le mode de l'assentiment à une proposition disant qu'il fait beau, ou, si l'on préfère, à une phrase traduisible par la phrase française "il faut beau" ${ }^{16}$.

Toutefois, Sperber fait remarquer que la notion d'attitude propositionnelle ne peut pas être reprise telle quelle par les ethnologues ou les historiens. En effet, écrit-il très justement, la communication ordinaire ne fonctionne pas, en général, comme l'exigerait la notion de croyance que les philosophes nous proposent.

Dans l'explication que donnent les philosophes, on a en quelque sorte deux étapes (logiques, pas chronologiques).

$1^{\circ}$ D'abord, une proposition est énoncée (elle est, si l'on veut, soumise à l'assentiment du sujet des croyances). A cette étape, la proposition est comprise dans sa signification. Or la signification d'une proposition est donnée par ses conditions de vérité : le sujet comprend la phrase "p" en saisissant quel est l'état du monde en vertu duquel la phrase "p" est vraie, dans le cas où cette phrase est vraie.

$2^{\circ}$ Ensuite, le sujet déclare s'il accepte ou non cette proposition (en vertu du contenu qu'il vient d'appréhender). S'il dit accepter la proposition que $\mathrm{p}$ (= la phrase "p" disant que p), nous disons qu'il croit que p. Voici pourquoi la croyance peut apparaître comme l'attitude que prend un sujet à l'égard d'un objet propositionnel.

Dans une communication réelle, il arrivera que le sujet accepte bien des propositions qu'il ne comprend qu'imparfaitement. On ne peut donc pas dire qu'il se prononce sur un énoncé dont il a pleinement saisi les conditions de vérité. D'où la question : dans ce cas, que croit-il ? Comment un ethnographe pourra-t-il spécifier le contenu des croyances d'un sujet moyen ? (Il suffit d'imaginer un sociologue des religions s'interrogeant sur les croyances

\footnotetext{
${ }^{16}$. A noter que, pour être une "représentation interne", cette phrase doit être mentale, c'est-à-dire qu'elle doit être formulée dans une "langue de la pensée".
} 
effectives des fidèles d'un culte dont les prêtres auraient développé la théologie en un système complexe de dogmes subtils.)

Sperber a entièrement raison de dire que la notion d'attitude propositionnelle ne paraît pas directement applicable dans les sciences sociales. Toutefois, ce qui m'intéresse ici n'est pas ce point (dont je reconnais qu'il est capital pour une philosophie des sciences sociales). C'est l'orthodoxie ontologique de la solution proposée. En effet, Sperber introduit une distinction entre deux sortes de croyances ${ }^{17}$.

Le mieux est de partir de l'exemple dont il l'illustre. Pierre, un lecteur peu averti des questions économiques, lit dans son journal (au milieu des années 1970) l'énoncé suivant : "la stagflation est devenue le premier problème des économies occidentales". Il le croit bien volontiers. Toutefois, nous supposons qu'il ne saisit pas pleinement le sens du mot "stagflation". Le journaliste qui a écrit cette phrase en connaissait les conditions de vérité : en écrivant cela, il a donc exprimé une croyance qui portait sur un objet propositionnel. En revanche, notre lecteur Pierre n'a pas une croyance de ce type, car l'objet de sa croyance n'est pas identifiable par les conditions de vérité de la proposition émise par le journaliste.

\begin{abstract}
Mais, alors, qu'est-ce au juste que Pierre croit ? Ce ne peut pas être la proposition exprimée par le journaliste, puisque Pierre ne dispose pas de tous les concepts nécessaires pour se la représenter. Ce n'est pas seulement l'énoncé que Pierre a lu dans son journal puisque Pierre pourrait exprimer sa croyance sans citer cet énoncé (...) Ce que croit Pierre semble donc être une représentation qui combine plusieurs concepts avec un terme dont il n'a pas complètement analysé le contenu conceptuel. (SA, p. 70; j'ajoute les italiques.)
\end{abstract}

Sur quoi porte donc la croyance de Pierre ? Puisque les conditions de vérité de l'énoncé du journaliste ne sont qu'incomplètement connues de Pierre, Sperber propose de dire que l'objet de la croyance de Pierre est une idée ou une pensée "semi-propositionnelle". Un même énoncé exprime la croyance du journaliste et celle de Pierre. Dans le cas du journaliste, cet énoncé exprime une croyance dont l'objet est une pensée propositionnelle (une idée bien comprise). Dans le cas de Pierre, cet énoncé exprime une croyance dont l'objet est une pensée semi-propositionnelle (une idée à demi comprise).

Sperber offre ici une comparaison qui éclaire bien les choses. Lorsque le lecteur croit quelque chose qu'il ne comprend pas pleinement, c'est comme s'il disposait d'une adresse incomplète. Cette adresse lui donne quelques indications, mais ne suffit pas à identifier un domicile et un seul. De même, une représentation semi-propositionnelle ne suffit pas à identifier une proposition.

\footnotetext{
17. Pour exposer cette distinction, je me tourne vers un passage d'un ouvrage antérieur du même auteur, $L e$ savoir des anthropologues (Paris, Hermann, 1982, p. 70-71), passage auquel Sperber lui-même nous renvoie (CI, p. 122). Le titre de ce livre sera abrégé SA.
} 
Une adresse individuelle, qui a pour fonction d'identifier un domicile et un seul, doit être complète. Si, par exemple, il manque le numéro de la rue, le domicile est localisé sans être pour autant identifié. De même, une représentation conceptuelle a pour fonction d'identifier une proposition et une seule. Mais une représentation conceptuelle peut être incomplète, c'est-à-dire comporter des éléments dont le contenu conceptuel n'est pas entièrement connu du sujet, et donc ne pas remplir tout à fait sa fonction. (SA, p. 71)

La solution avancée par Sperber permet, comme c'est son but, de tenir compte de la différence entre les croyances du sage et la foi du charbonnier. Le sage donne son assentiment à des propositions. Il est comme quelqu'un qui possède l'adresse correcte et complète du domicile auquel il doit se rendre. Le charbonnier donne son assentiment à des choses qu'il a entendu dire, sur la foi d'autrui. Il est comme quelqu'un qui possède une adresse incomplète, une adresse qui ne détermine pas un domicile et un seul, mais plutôt un ensemble de domiciles (un ensemble d'interprétations), l'ensemble de tous les domiciles qu'on peut déterminer selon la façon dont on complète les indications fournies.

Il y a toutefois un prix à payer pour cette réforme de la notion philosophique d'attitude propositionnelle. Nous devons maintenant distinguer entre une proposition et la représentation d'une proposition, tout comme on distingue entre une adresse et ce dont l'adresse est l'adresse (le domicile). Comme le fait justement remarquer Sperber, on ne saurait confondre une adresse et un domicile :

On remarquera que parler de représentations semi-propositionnelles n'oblige pas à admettre l'existence de "semi-propositions" (de même qu'on peut parler d'adresses incomplètes sans admettre l'existence de semi-domiciles). (SA, p. 85, note 29)

Autrement dit, le concept de représentation s'est dédoublé.

$1^{\circ}$ Dans un premier sens, la représentation est l'objet du croire, elle est ce que croit le sujet croyant. Le programme cognitiviste auquel se référait Sperber était apparemment de tenir la relation du sujet à l'objet de croyance pour une relation entre un être humain (organisme) et une "représentation interne", donc un objet mental susceptible d'être redécrit comme objet cérébral, donc "de ce monde". Sperber citait Fodor : "avoir une attitude propositionnelle, c'est être dans une attitude computationnelle à une représentation interne" (cité SA, p. 70).

$2^{\circ}$ Dans un deuxième sens, la représentation est celle que le sujet peut se faire, en fonction de ses ressources conceptuelles, d'une proposition : il se la représente adéquatement ou non. Ainsi, Pierre n'a pas les moyens nécessaires pour se représenter la proposition du journaliste. L'objet du croire est comme le domicile, mais ce domicile doit être représenté au sujet par une adresse, laquelle sera complète ou incomplète (un domicile ne saurait être incomplet). 
Dans ces conditions, les propositions dont nous parle la doctrine des attitudes propositionnelles cessent de coïncider avec des représentations présentes dans les têtes des sujets croyants. Dans ces têtes, il n'y a que des adresses. Les propositions redeviennent des objets auxquels les sujets se rapportent par le moyen des représentations qu'ils s'en font. De sorte que se pose à nouveau le vieux problème de l'identité de ces objets propositionnels ${ }^{18}$. On peut bien, en les comparant, voire si deux adresses sont ou non deux versions d'une seule et même formule prétendant identifier un domicile. Mais comment pourra-t-on, rien qu'en considérant une adresse, savoir si elle identifie un domicile et un seul ? Comment savoir s'il ne manque pas la mention du bâtiment, de l'escalier, de l'étage, etc. ? Comment pourrait-on savoir, rien qu'en comparant deux adresses, qu'elles ne correspondent pas au même domicile (dès lors que, dans les conditions réelles de la communication humaine, nous n'avons par ailleurs la garantie que soit respectée la règle d'un bon système des adresses : pour toute adresse, un domicile et un seul, et, pour tout domicile, une adresse en circulation et une seule) ?

Autrement dit, les propositions, puisqu'elles sont accessibles à travers des "adresses", sont des choses qu'il faut se représenter et non pas seulement des choses qui représentent elles-mêmes autre chose (à savoir des états du monde). Bref, Sperber se trouve à nouveau devant le problème des platoniciens : comment établir une relation entre nous-mêmes et les significations en soi (les propositions en bonne et due forme, objets bien identifiés dans les croyances bien comprises et objets vaguement identifiés dans les croyances mal comprises) ? On n'a pas de critères d'identité pour de tels objets propositionnels, qui sont nécessairement (dans une telle optique) des objets abstraits.

\section{Langues et discours}

Considérons maintenant les entités linguistiques. Dans l'optique d'une ontologie moniste, on peut concevoir le principe d'un inventaire de telles entités. Est-ce qu'un tel inventaire va comporter des entités telles que le français, l'allemand, le chinois ? Si c'était le cas, il faudrait "accepter dans son ontologie" ces représentations culturelles que sont les langues.

D'après Sperber, il n'y aura pas de tels articles dans l'inventaire, ou, du moins, il n'y en aura pas de façon irréductible. Un inventaire "ultime" des entités linguistiques n'aurait pas à mentionner les langues (objets abstraits) puisqu'il aurait déjà enregistré l'existence des discours individuels (objets concrets). En effet, si nous comptons les phrases produites par les individus, nous comptons du même coup les entités réelles que nous pouvons "représenter"

\footnotetext{
${ }^{18}$. Sur l'histoire et la logique de ce problème, voir par exemple Arthur Prior, Objects of Thought (Oxford U.P., 1971) et The Doctrine of Propositions and Terms (Londres, Duckworth, 1976).
} 
par ailleurs par l'artifice d'une hypostase de la langue. Il en va ici comme des croyances partagées. Si j'ai déjà rencensé, parmi les entités, les croyances de Paul et de Pierre, il n'y a plus lieu de mentionner la "croyance partagée" par Paul et Pierre (puisqu'elle n'est rien d'autre que la ressemblance entre la croyance de l'un et la croyance de l'autre).

Je me propose de soutenir, moi aussi, qu'aucun inventaire des "entités linguistiques" ne va contenir à la fois des discours effectifs et des langues. Mais la raison ne sera pas celle de Sperber. Elle ne sera pas qu'un démographe ne va pas compter le Français moyen comme une unité s'ajoutant aux personnes recensées. La raison sera que les discours et les langues n'entrent pas dans la même catégorie.

Il est vrai qu'on parle parfois des langues comme si elles étaient des sujets d'activités historiques. On usera par exemple de formules de ce genre : le français recule en Afrique, il perd des points (ou il en gagne) en Amérique latine, il s'enrichit, il se dégrade. De telles hypostases sont anodines. Personne ne doute qu'elles puissent être éliminées : lorsque le français perd du terrain, cela veut dire qu'il y a moins de gens qui savent le français ou qui l'utilisent dans une aire donnée.

Toutefois, la langue traitée comme une hypostase, comme un être qui aurait une vie, des aventures, ne ressemble pas à l'objet abstrait dont nous parle Sperber. D'ailleurs un objet abstrait ne saurait avoir une histoire ou des aventures empiriques.

Sperber a expliqué sur un mode général comment produire une hypostase à partir de divers individus concrets. Il a illustré la production d'une telle hypostase par l'exemple d'une croyance folklorique sur les sorcières. Mais passer des croyances de Paul et Pierre à une "croyance partagée", c'est passer de ce que croient telle et telle personnes (qu'on nomme) à ce qu'on croit, "on" valant ici comme un pronom indéfini susceptible d'être remplacé par une liste des croyants (Paul, Pierre, etc.) ${ }^{19}$.

Supposons que nous procédions ainsi en considérant, non plus les croyances de Paul et Pierre, mais les paroles de Paul et Pierre. Le résultat sera d'échanger ce que disent Paul et Pierre contre une abstraction : ce qu'on dit. Nous aurons engendré par hypostase une parole moyenne, un discours commun. Cette hypostase n'est donc pas du tout une langue commune. La question reste en effet posée de déterminer en quelle langue ce discours moyen est tenu.

Sperber s'est donné pour but d'expliquer que le statut ontologique d'une langue (comme le français) est celui d'un objet abstrait. Or il ne nous parle pas d'une langue, mais d'un ensemble de phrases. Il a donc fait de la langue un discours : et c'est justement cela qui nous embarrasse.

Mais, si la langue n'est pas une simple image moyenne des discours effectivement tenus, alors, qu'est-ce que c'est ? Ici, le partisan de la théorie mentale des signes objectera

\footnotetext{
${ }^{19}$. D'être "en gros" remplacé, c'est-à-dire en faisant "abstraction" des différences individuantes.
} 
qu'il ne voit pas ce qu'on lui demande d'ajouter aux paroles. Lorsque je fait référence à la langue française, est-ce que je prétends poser l'existence d'une entité distincte des paroles prononcées ou écrites que nous comptons comme du français?

La réponse à cette objection est qu'il faut sortir de la perspective de la liste unique des entités pour reconnaître, comme nous devons le faire, la différence entre une langue et un discours. Qu'il s'agisse d'une différence de catégorie, cela veut dire qu'on ne saurait individuer indifféremment des paroles et des langues (les compter sur la même liste). Une chose est de recenser les paroles produites (par exemple au cours d'une réunion), autre chose est de recenser les langues parlées (au cours de la même réunion).

Pour apprécier le bien-fondé de cette différence de catégorie, il convient d'abord de voir où est l'erreur dans la confusion entre une langue et un discours.

Dans un exposé portant sur les notions de signification et d'usage ${ }^{20}$, Gilbert Ryle avait fait observer que l'adjectif "linguistique" était en réalité ambigu puisqu'il pouvait renvoyer aussi bien à la langue qu'au discours. On ajoutera que le mot anglais "language" comporte la même ambiguïté (de façon beaucoup plus marquée que son parent en français), et que cette ambiguïté n'est pas sans obscurcir bien des discussions qu'ont en anglais les philosophes du langage.

Ryle reprenait en fait la distinction saussurienne de la langue (institution sociale) et de la parole (acte personnel). Cette distinction, il ne la tenait pas directement du Cours de linguistique générale, mais de l'ouvrage d'inspiration saussurienne d'Alan Gardiner intitulé Théorie du discours et de la langue ${ }^{21}$. Il faut d'ailleurs avouer que l'opposition en question trouve chez Gardiner une clarté philosophique qui lui manquait dans bien des exposés saussuriens.

On méconnaît la différence de catégorie qu'il y a entre un discours (speech) et une langue (language) chaque fois qu'on essaie de définir une langue, par exemple le français, comme un ensemble ou une totalité de phrases (ce qui est malheureusement la définition favorite des philosophes analytiques).

Si le français se définit comme un ensemble de phrases, une question se pose : veut-on parler de l'ensemble des phrases effectivement produites en français ou de l'ensemble des phrases possibles ? Dans les deux cas, on a des conséquences indésirables. Si le français est l'ensemble des phrases effectivement produites dans cette langue, on a cette conséquence : chaque fois qu'une phrase nouvelle est prononcée ou écrite en français, chaque fois donc que le corpus des phrases effectives s'enrichit d'un exemplaire différent quant au type, la langue française est enrichie, donc modifiée. Chaque locuteur, dès qu'il parle, ajoute une phrase au corpus. Par là même, de façon plus ou moins grave, il modifie le sens de l'ensemble des

\footnotetext{
${ }^{20}$. "Use, Usage and Meaning" (1961), republié dans : G.H.R. Parkinson (éd.), The Theory of Meaning, Oxford University Press, 1968.

${ }^{21}$. Alan Gardiner, The Theory of Speech and Language, Oxford U.P., 1932; 2e éd. 1951.
} 
phrases du corpus. On a donc la conséquence herméneutique : le sens des écrits passés ne cesse de changer historiquement, du seul fait de l'évolution de la langue comprise comme ensemble du discours. Il y a par conséquent une histoire du sens de ce qu'a dit tel auteur, histoire qui est celle des lectures ou des "réceptions" du texte à telle ou telle époque. Le sens de Ronsard et de La Fontaine n'est pas le même avant et après Victor Hugo.

Sartre a poussé jusqu'aux dernières conséquences cette forme de "holisme" malencontreux. Quand je parle, dit-il, je modifie le sens de la langue et donc le sens des paroles des autres. Du même coup, tant que je ne suis pas le dernier à parler, le sens de mes paroles est destiné à m'être dérobé par l'intervention des locuteurs qui s'expriment dans ma langue après moi.

Supposons maintenant que la langue française, ce soit l'ensemble des phrases possibles dans cette langue, l'ensemble des expressions bien formées en vertu de certains axiomes ou de certaines règles. Dans ce cas, que devient l'histoire de la langue française ?

Une distinction s'impose donc. Reste à voir en quel sens elle est une distinction de catégorie. Pour cela, nous pouvons justement nous tourner vers l'exposé d'A. Gardiner.

Nous apprenons les mots d'une langue, c'est-à-dire que nous apprenons comment les utiliser. Lorsque nous les utilisons, nous produisons (construisons) des phrases. Si quelqu'un n'émettait jamais que des phrases qu'il aurait apprises toutes faites, nous dirions assurément qu'il ne sait pas réellement la langue utilisée, même s'il parvenait à se débrouiller dans quelques situations élémentaires. Ainsi, le locuteur est l'auteur de son discours (puisqu'il construit lui-même ses phrases), mais il n'est pas, en général, l'auteur de ses mots ou de sa syntaxe.

Nous avons bien deux catégories. D'un côté, ce qui relève d'un savoir, donc d'une capacité du sujet, capacité qui a été acquise (par l'apprentissage de la langue), qui est plus ou moins ancienne, plus ou moins souvent exercée par ce sujet. De l'autre côté, une activité de parler (act of speech). On n'a pas la même sorte d'unité ou d'entité d'un côté et de l'autre. En d'autres termes, on ne peut pas demander combien d'entités linguistiques il y a dans une énonciation, alors qu'on peut demander, d'une part, combien il y a de mots, et d'autre part, combien il y a de phrases.

Gardiner fait à cet égard une démonstration convaincante en considérant le cas des actes de parole qui, à la façon des interjections, ne font usage que d'un seul mot. Dans son exemple, un locuteur (John) regarde par la fenêtre et s'adresse à une interlocutrice (Mary) qui se trouve à côté de lui en lui disant : "Rain ! " 22 . Combien d'unités linguistiques trouve-t-on dans cet acte de parole ? Gardiner explique qu'on doit distinguer ici deux sortes d'unités linguistiques.

Les unités du discours (units of speech) sont les phrases. Les unités de la langue (units of language) sont les mots. Par conséquent, on ne peut pas compter des "unités

\footnotetext{
${ }^{22}$. Il faudrait fabriquer un exemple différent en français.
} 
linguistiques" sans plus, mais seulement des mots (dans le vocabulaire d'une phrase, d'un livre, d'une époque) ou des phrases (dans un discours). Combien faut-il de mots à John pour s'exprimer ? Combien lui faut-il de phrases?

Il est remarquable que Gardiner soit parfaitement conscient de poser ici un problème ontologique (op. cit., p. 88-89). James dit à Mary : "Rain !" On ne trouve qu'un seul mot dans cette phrase. Dira-t-on qu'il y a une entité ou deux ? A première vue, écrit-il, on a envie de dire qu'il n'y a qu'une entité, et que les termes de "mot" et de "phrase" ne sont pas ici des termes pour deux entités distinctes (names of separate and overlapping entities). L'esprit d'économie conduirait donc à dire que "mot" et "phrase" sont des termes pour deux aspects distincts d'une seule et même entité (names of distinct aspects of one and the same entity). Et pourtant, conclut Gardiner, il y a bien en réalité deux choses à considérer, pas deux aspects d'une même chose. Dans l'échange en question, il y a bien un mot et un seul, mais il y a également une phrase. Toutefois, ces choses ne sont pas dans la même catégorie, de telle sorte qu'on se gardera de dire : il y a, outre le mot, la phrase (à la façon dont on dirait à juste titre que dans l'exclamation "la porte !", on trouve, outre le substantif, l'article défini).

Pour faire apparaître la différence réelle entre mot et phrase, il suffit de poser ces deux questions, dont on voit tout de suite qu'elles ne se posent, chacune de son côté, que pour l'une ou l'autre des deux catégories :

$1^{\circ}$ Quel est l'âge du mot?

$2^{\circ}$ Quel est l'auteur de la phrase ?

Le mot est un article de vocabulaire. Sa date de naissance est parfois connue. Les dictionnaires indiquent, avec plus ou moins de précision, son âge. En général, on sait d'où vient le mot (quels sont ses ancêtres). Mais que pourrait bien signifier une recherche sur la provenance de la phrase de James (à moins de supposer qu'il répète une phrase, qu'il l'emprunte à quelqu'un) ? On peut se demander quand est apparu, dans l'histoire de la langue anglaise, le mot "rain". Mais l'âge du mot "rain" n'est pas l'âge de la phrase de James à Mary. Cette phrase a un auteur, mais n'a pas longtemps un âge. Elle atteint vite l'âge qu'il lui faut pour parvenir à sa destinataire Mary. En effet, les phrases, le plus souvent, ne sont pas destinées à durer. Ce sont, dit Gardiner, des constructions éphémères, des constructions ad hoc, qu'on met sur pied à telle occasion et qu'on défait ensuite (op. cit., p. 90).

On pourrait dire que la typographie marque la différence de catégorie entre le mot et la phrase. Dans le dictionnaire, nous avons seulement le mot "rain". Dans le petit récit inventé par Gardiner, il s'agit d'autre chose, à savoir du mot "rain" suivi d'un point d'exclamation.

\section{Conclusion}


Si nous distinguons les catégories selon le mode d'identification, rien ne nous empêche plus de dire qu'il y a dans le monde des langues (puisque cela ne nous impose plus de chercher des objets qui soient ces langues). Il n'y a donc aucune raison de ne pas dire que deux personnes qui parlent en français et qui sont les auteurs de leurs phrases se comprennent parce qu'elles comprennent l'une et l'autre le français.

Soit deux personnes remplissant deux conditions :

$1^{\circ}$ elles se parlent en français ;

$2^{\circ}$ elles sont les auteurs de leurs phrases.

Rien ne s'oppose à ce que nous expliquions ainsi le fait de leur communication : ces deux personnes se comprennent parce qu'elles comprennent l'une et l'autre le français.

Ce qui nous évite d'avoir à nous préoccuper des divers paradoxes de la communication précaire, c'est donc la référence à l'institution de la langue. Dans une théorie individualiste et mentaliste du langage, il est dans la nature du langage de rendre la communication malaisée, jamais assurée. Dans une théorie holiste et anthropologique du langage, il est dans la nature du langage de rendre la communication possible, pourvu qu'on veuille bien apprendre la langue de ses interlocuteurs. 